\title{
Keratin 6, induced by chronic cisplatin exposure, confers chemoresistance in human gastric carcinoma cells
}

\author{
SUNG-CHUL LIM ${ }^{1,2}$, KESHAB RAJ PARAJULI ${ }^{2}$ and SONG IY HAN SN, $^{2,3}$ \\ ${ }^{1}$ Department of Pathology, ${ }^{2}$ Research Center for Resistant Cells, and ${ }^{3}$ Division of Premedical Science, \\ College of Medicine, Chosun University, Gwangju 61501, Republic of Korea
}

Received January 29, 2019; Accepted June 7, 2019

DOI: 10.3892/or.2019.7201

\begin{abstract}
Currently, various types of keratins have been reported to be highly expressed in cancer cells and to be associated with a malignant phenotype. In the present study, it was found that expression levels of keratin 6 (K6), keratin 16 (K16), and keratin 17 (K17) were highly elevated in SNU601 cells resistant to cisplatin (SNU601-cis2 and SNU601-cis10), but not in the parental SNU601 cells as confirmed by quantitative PCR, immunoblotting, and immunofluorescence assays. K6 is a type II keratin and is known to form a keratin filament in conjugation with type I keratin, K16 or K17. Thus, we attempted to understand the role of the overexpression of $\mathrm{K} 6 / \mathrm{K} 16$ or $\mathrm{K} 6 / \mathrm{K} 17$ keratin filaments by regulating the expression of K6. Silencing of K6 by siRNA in SNU601-cis2 cells promoted oxaliplatin-induced apoptosis in the resistant cells as shown by increased apoptotic body formation, caspase- 8 and caspase-3 cleavage, and cytochrome $c$ release. In addition, induction of K6 levels in wild-type SNU601 cells, by transfection with pCMV6-K6A and pCMV6-K6B overexpression vectors, resulted in decreased apoptosis in response to cisplatin and L-OHP. Platinum drugs, such as oxaliplatin, were shown to induce the extrinsic apoptotic pathway by inducing lipid raft formation and death receptor recruitment into lipid rafts. However, in the resistant cells, the oxaliplatin-triggered extrinsic apoptotic pathway appeared to be suppressed by decreased lipid raft formation, and recruitment of death receptor 5 and FADD into lipid rafts. Therefore, the increase in the levels of the K6 filament may be associated with the regulation of lipid raft formation and may contribute, at least in part, to resistance to anticancer drugs.
\end{abstract}

Correspondence to: Professor Song Iy Han, Division of Premedical Science, College of Medicine, Chosun University, 309 Pilmun-daero, Dong-gu, Gwangju 61501, Republic of Korea

E-mail: sihan@chosun.ac.kr

Key words: keratin 6, cisplatin, drug resistance, apoptosis, gastric cancer

\section{Introduction}

Gastric cancer is often diagnosed at an advanced stage and it commonly recurs even after complete resection. Thus, chemotherapy is required for the treatment of unresectable cases and prevention of cancer recurrence. However, consistent exposure to chemotherapeutics often results in drug resistance, which is a serious impediment to cancer treatment. Hence, research has been conducted to reveal the mechanism of cancer chemoresistance to overcome this problem.

The development of drug resistance is a complex process due to various mechanisms. These include overexpression of efflux pumps, including ABCC, upregulation of survival pathways, elevation of the antioxidant system, defects in apoptotic signaling, and alterations in the dynamics of cytoskeletons (1-4). The cytoskeleton is one of the main targets for cancer treatment, since the cytoskeletal network plays pivotal roles in cytokinesis, mitosis, membrane transport, and many other processes essential for rapid growth and metastasis of cancer cells. The roles of actin and microtubule cytoskeletons are relatively well investigated in cancer biology, and many efforts have been made to develop therapeutic strategies targeting them in carcinomas (5-7). Recently, it was found that certain keratin molecules interact with multiple factors that are involved in cellular survival processes and are associated with cancer cell malignancy and resistance to apoptosis. Several of them are recognized as markers for tumor prognosis as well as for tumor diagnosis.

The keratin filament is a $10-\mathrm{nm}$ thick intermediate filament that belongs to the three major cytoplasmic filament networks, together with microfilaments and microtubules. Keratins are mainly found in the outer layer of the skin, hair, and nails, and they provide mechanical strength and protection against various external stimuli, including heat, UV, septic infection, and physical stress $(8,9)$. In contrast to microtubules and microfilaments that are uniform, there are diverse types of keratin filaments composed of different isoforms of keratin proteins. The keratin gene family consists of 54 genes in humans, which are either found as the acidic type I or the basic or neutral type II keratin genes (28 type I genes, 26 type II genes). Complete keratin filaments are assembled as heteropolymers by non-covalent interaction between type I and type II keratin chains, and expression of keratin proteins is also paired with acidic and basic keratins in a tissue- and differentiation-specific fashion (10-13). Since different keratin pairs are present in 
different regions or different developmental stages of epithelia, the regulation of their cell type- and stage-specific expression pattern may be strictly associated with their own unique function. Indeed, recently identified results indicate that keratin filaments are involved in the regulation of various cellular functions including cell survival, differentiation and transformation, by influencing intracellular signaling. For instance, K8 expression correlates with c-FLIP level and ERK1/2 signaling in epithelial cells and protects cells from death ligand-induced apoptosis (14). K8/K18 influences insulin receptor signaling via modulation of phosphoinositide-dependent Akt and Rab5 signaling in hepatocytes (15), and is also implicated in colonic epithelial cell differentiation through regulation of the Notch1 signaling (16). K5/K14 is involved in the mediation of Tap63 and Notch-1 signaling during cell transformation (17). Furthermore, elevated expression of certain keratins, such as $\mathrm{K} 8, \mathrm{~K} 17, \mathrm{~K} 18$ and $\mathrm{K} 20$ is shown to be linked to malignant transformation and unfavorable prognosis of various types of cancer (18). In addition, primary breast cancer exhibits an altered expression profile of keratin during metastatic progression, and K81, known as hair keratin, which is not found in normal epithelial cells, is expressed in breast cancer cells, where it was found to be involved in metastasis. In this study, it was demonstrated that keratin 6 (K6) is extremely overexpressed in cisplatin-resistant variants of human gastric cancer SNU601 cells, and we investigated the role of $\mathrm{K} 6$ in drug responsiveness.

\section{Materials and methods}

Cell culture and drug treatment. Human gastric cancer SNU-601 cells were obtained from the Korean Cell Line Bank (Seoul, Korea), and the cisplatin-resistant sublines (SNU601-cis2 and SNU601-cis10), selected by gradually increasing cisplatin concentrations from 2 to $10 \mu \mathrm{g} / \mathrm{ml}$, were a gift from Professor C. H. Choi of the Department of Pharmacology, Chosun University, Korea (19). The cells were cultured in RPMI-1640 medium (Invitrogen; Thermo Fisher Scientific, Inc.) supplemented with $10 \%$ (v/v) fetal bovine serum and $1 \%$ penicillin-streptomycin at $37^{\circ} \mathrm{C}$ in an atmosphere containing $5 \% \mathrm{CO}_{2}$. Oxaliplatin (L-OHP) was obtained from Boryung Pharmaceutical (Seoul, Korea), and cisplatin (CDDP) was purchased from Sigma-Aldrich; Merck KGaA. Unless specified otherwise, drugs were purchased from Calbiochem; EMD/Merck KGaA.

Analysis of apoptosis. Treated cells were stained with $1 \mu \mathrm{g} / \mathrm{ml}$ Hoechst 33342 (HO) for $15 \mathrm{~min}$, and both suspended and attached cells were collected and centrifuged. The pooled cell pellets were washed and fixed in $3.7 \%$ formaldehyde, and an aliquot of the suspension was centrifuged at $600 \mathrm{x} \mathrm{g}$ for 10 min in a Cytospin centrifuge (Shandon, Thermo Fisher Scientific, Inc.). Slides were prepared, air-dried, mounted in anti-fade solution, and observed under a fluorescence microscope (DM5000, Leica, Germany) using excitation and emission wavelengths of 340 and $425 \mathrm{~nm}$, respectively. Condensed or fragmented nuclei were considered indicative of apoptosis. A total of 500 cells distributed across random microscope fields of view were counted, and the number of apoptotic and non-apoptotic cells was expressed as a percentage of the total.
Immunoblotting. Treated cells were lysed in a lysis buffer (50 mM HEPES, $150 \mathrm{mM} \mathrm{NaCl,} \mathrm{1 \%} \mathrm{Triton} \mathrm{X-100,} 5 \mathrm{mM}$ EGTA, and protease inhibitor cocktail) and equal amounts of protein extracts were electrophoretically separated using 10-12\% SDS-PAGE and transferred to a nitrocellulose membrane using a standard technique. Antibodies were used to probe for K6 (dilution 1:1,000; cat. no. sc-22479; Santa Cruz Biotechnology), K16 (dilution 1:1,000; cat. no. sc-53255; Santa Cruz Biotechnology), K17 (dilution 1:500; cat. no. sc-393002; Santa Cruz Biotechnology), pan-keratin (dilution 1:1,000; cat. no. sc-8018; Santa Cruz Biotechnology), cytochrome $c$ (dilution 1:500; cat. no. sc-13156; Santa Cruz Biotechnology), pro-caspase-8 (dilution 1:500; cat. no. sc-73526; Santa Cruz Biotechnology), caveolin-1 (dilution 1:500; cat. no. sc-894; Santa Cruz Biotechnology), Fas associated protein with death domain (FADD) (dilution 1:500; cat. no. sc-13156; Santa Cruz Biotechnology), $\beta$-actin (dilution 1:1,000; cat. no. sc-8432; Santa Cruz Biotechnology), cleaved caspase-3 (cysteinyl aspartate-specific protease-3; dilution 1:500; cat. no. 9661; Cell Signaling Technology) and DR5 (TRAIL-R2; dilution 1:500; cat. no. 2019; ProSci). As second antibodies, anti-rabbit HRP (dilution 1:3,000; cat. no. sc-2030; Santa Cruz Biotechnology), anti-mouse HRP (dilution 1:3,000; cat. no. sc-2031; Santa Cruz Biotechnology) and anti-goat HRP (dilution 1:2,000; cat. no. sc-2020; Santa Cruz Biotechnology) were used. Anti-a-tubulin (dilution 1:2,000; cat. no. B-5-1-2; Invitrogen; Thermo Fisher Scientific, Inc.) was used as a loading control. Signals were acquired using an Image Station 4000MM image analyzer (Kodak).

RNA interference (RNAi). For the RNAi experiment, the small interfering RNA (siRNA) of K6 \#1 was purchased from Bioneer (Daejeon, Korea) and the siRNA of K6 \#2 was purchased from Ambion (Thermo Fisher Scientific, Inc.). For the control siRNA, 5'-CCUACGCCACCAAUUUCGU(dtdt)-3' (sense) and 5'-ACGAAAUUGGUGGCGUAGG(dtdt)-3' (antisense) were used. Cells were individually transfected with siRNA oligonucleotides using an Amaxa Transfection System $^{\mathrm{TM}}$ (Basel, Switzerland) and grown for $24 \mathrm{~h}$ prior to drug treatment.

Real-time reverse transcription-polymerase chain reaction (PCR). Real-time PCR was performed with the Light Cycler 2.0 (Roche, Switzerland) using the Fast Start DNA Master SYBR-Green I Kit (Roche). For verification of the correct amplification product, PCR products were analyzed on a $2 \%$ agarose gel stained with ethidium bromide. The sequences of the primers were as follows: For $\beta$-actin, 5'-GAC TATGACTTAGTTGCGTTA-3' and 5'-GCCTTCATACAT CTCAAGTTG-3'; for K6A, 5'-TTCAGAACAACTTCCACT TACTTTCC-3' and 5'-GTCACTTGTGCTTTCATGGATACT G-3'; for K6B, 5'- GTAAAACGACGGCCAGT-3' and 5'-TAA TACGACTCACTATAGG-3'; for K6C, 5'-GTAAAACGA CGGCCAGT-3' and 5'-TAATACGACTCACTATAGG-3'; for K16, 5'-AAGGAGGAGCTGGCCTA-3' and 5'-CTCTGCCAT CTGCTCGTA-3'; and for K17, 5'-CATGCAGGCCTTGGA GATAGA-3' and 5'-CACGCAGTAGCGGTTCTCTGT-3'. PCR was conducted at $95^{\circ} \mathrm{C}$ for $10 \mathrm{~min}$, followed by 45 cycles of $95^{\circ} \mathrm{C}$ for $15 \mathrm{sec}, 60^{\circ} \mathrm{C}$ for $20 \mathrm{sec}$, and $72^{\circ} \mathrm{C}$ for $21 \mathrm{sec}$. Melting curve analysis was performed to confirm production 
of a single product. Negative controls without template were included for each run. Data were analyzed using Light Cycler software version 4.0 (Roche). Relative gene expression was calculated by the $2^{-\Delta \Delta \mathrm{Cq}}$ method (20).

Confocal microscopy. To detect keratins, cells grown on coverslips were fixed with $4 \%$ paraformaldehyde for $10 \mathrm{~min}$, permeabilized with $2 \%$ Triton $\mathrm{X}-100$ for $10 \mathrm{~min}$, and blocked with $5 \%$ BSA/PBS for $30 \mathrm{~min}$. Then, the coverslips were incubated with primary antibodies and reacted with fluorescein isothiocyanate (FITC)-conjugated secondary antibody [anti-mouse FITC (dilution 1:100; cat. no. sc-2010; Santa Cruz Biotechnology) and anti-goat FITC (dilution 1:100; cat. no. sc-2024; Santa Cruz Biotechnology)]. To visualize lipid rafts, cells grown on coverslips were fixed with $1.5 \%$ paraformaldehyde for $5 \mathrm{~min}$ at $-20^{\circ} \mathrm{C}$ and stained with $10 \mu \mathrm{g} / \mathrm{ml}$ FITC-labeled cholera toxin B (CTxB). After washing, drying, and mounting, the prepared slides were observed under a laser-scanning confocal microscope (x600 magnification; FV1000; Olympus Optical Co.). Using an argon laser, FITC was excited at $488 \mathrm{~nm}$, and the evoked emission was filtered with a $515 \mathrm{~nm}$ band-pass filter.

Lipid raft isolation. Lipid rafts were isolated using sucrose density-gradient centrifugation. A total of $10^{8}$ cells were lysed for $30 \mathrm{~min}$ in $1 \mathrm{ml}$ lysis buffer (1\% Brij35 in HEPES buffer; $25 \mathrm{mM}$ HEPES, $1 \mathrm{mM}$ EDTA, and $150 \mathrm{mM} \mathrm{NaCl}, \mathrm{pH}$ 6.5) supplemented with a protease inhibitor cocktail and homogenized with a glass Dounce homogenizer. The homogenates were mixed with $1 \mathrm{ml}$ of $80 \%$ sucrose in HEPES buffer and placed at the bottom of a centrifuge tube. The samples were then overlaid with $6.5 \mathrm{ml}$ of $30 \%$ sucrose and $3 \mathrm{ml}$ of $5 \%$ sucrose, and centrifuged at $188,000 \mathrm{x}$ g for $18 \mathrm{~h}$ at $4^{\circ} \mathrm{C}$. Fractions $(1 \mathrm{ml})$ were collected from the bottom to the top of the gradient, and rafts were determined by measurement of the total cholesterol level. Fractions 3 through 5 of the sucrose gradients were pooled as the raft fraction; the rest was used as the non-raft fraction.

Stable gene expression. pCMV6-Entry, pCMV6-K6A, and pCMV6-K6B vectors were purchased from OriGene. These vectors were transfected individually into SNU601 cells using Amaxa Transfection System ${ }^{\mathrm{TM}}$. Stably transfected cells were selected and maintained in $200 \mu \mathrm{g} / \mathrm{ml} \mathrm{G} 418$ containing growth media.

Statistical analysis. All numerical data are presented as the mean \pm SE. All data represent the results of at least three independent experiments. Student's t-test was used for simple comparison, and one-way ANOVA with Tukey's test was applied to multiple comparisons to analyze differences in gene silencing or gene overexpression. A significant difference was assumed at $\mathrm{P}<0.05$.

\section{Results}

K6, K16, and K17 expression is elevated in the cisplatinresistant SNU601 cell sublines. It has been reported that human cancer cells that have developed resistance to cisplatin exhibit cross-resistance to various anticancer agents $(21,22)$.
Consistent with this result, the cisplatin-resistant variants, SNU601-cis2 and SNU601-cis10, which were previously developed by culturing SNU601 human gastric cancer cells in 2 and $10 \mu \mathrm{M}$ cisplatin, respectively, demonstrate a strong resistance to a wide range of cytotoxic agents apart from cisplatin (19). This is probably due to changes in the expression of a variety of genes involved in multiple pathways related to cell survival during the process of acquiring chemoresistance by cancer cells. It has been known that cytoskeletal protein plays an important role in the survival and protection of cancer cells against apoptotic stimuli, and has been used as a target for anticancer treatment for a long time. Thus, we investigated whether there was a change in the expression profile of intracellular cytoskeletal filaments in the chemoresistance-acquired cells. As shown in Fig. 1A, there was no change in the expression level of tubulin or actin; however, notably, the expression levels of K6, K16, and $\mathrm{K} 17$ in the keratin, constituting the intermediated filament, were found to be highly increased in both SNU601-cis2 and SNU601-cis10 resistant cells as detected by immunoblot assay. However, there was no difference in the detection of total Ks as recognized by the antibody against pan-K, suggesting that there was no significant difference in the overall level of keratin expression. This result was also confirmed by immunofluorescence assay using confocal microscopy (Fig. 1B). Subsequently, the mRNA levels of K6A (KRT6A), K6B (KRT6B), K6C (KRT6C), K16 (KRT16) and $K 17$ (KRT17) and genes were determined by real-time quantitative PCR. Consistently, mRNA levels of these genes were highly increased in the SNU601-cis2 and SNU601-cis10 resistant cells (Fig. 2) indicating that expression of these proteins is upregulated at the transcriptional level.

Knockdown of K6 partially restores drug sensitivity in the cisplatin-resistant SNU601 cells. In the present study, we explored the significance of the marked increase of a particular set of keratins in the drug-resistant SNU601 sublines. It was initially suspected that keratin overexpression is likely to contribute to the resistance of cells to cytotoxic stimuli. Here, we performed the gene interference study to examine whether the most highly induced keratin, K6, was associated with the drug-resistant phenotype of SNU601-cis2 (C2) cells. The cells were transfected with two different K6-specific siRNAs (K6RNAi \#1 and K6RNAi \#2) and treated with L-OHP, and then, apoptotic sensitivity was assessed. The silencing effect was confirmed by the decreased expression of $\mathrm{K} 6$ as determined by immunoblot assay (Fig. 3A). The silenced cells were treated with L-OHP, a platinum drug having stronger anticancer effect compared to cisplatin, and stained with Hoechst 33342. Then, fragmented or condensed apoptotic bodies were detected under a fluorescence microscope (Fig. 3B). As shown in Fig 3B, treatment with L-OHP slightly increased the apoptotic body formation in both K6 siRNA transfected cell lines compared to the control RNA-transfected cells (C2/CTLRNAi). The apoptotic body counts showed that silencing of K6 using both K6RNAi \#1 and K6RNAi \#2 significantly increased the percentages of apoptotic bodies after treatment with 50 and $100 \mu \mathrm{M}$ L-OHP, although the percentage of apoptotic bodies induced by L-OHP was much lower than that in the WT cells 
A

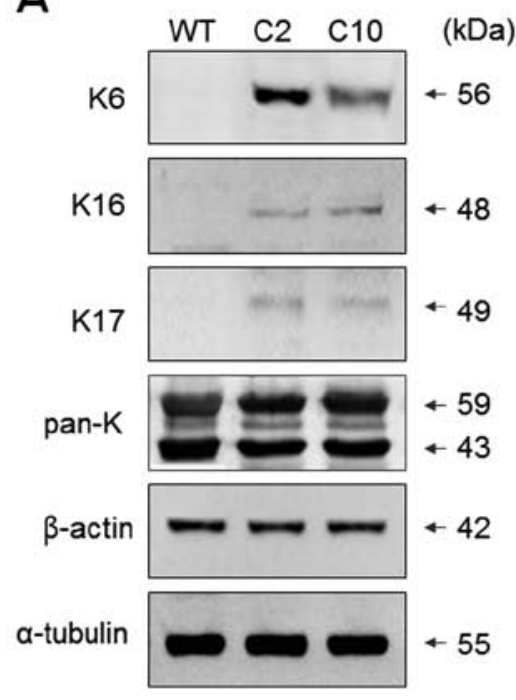

B
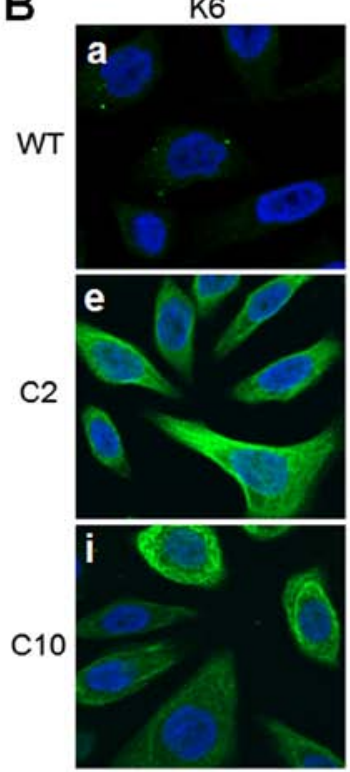

K16
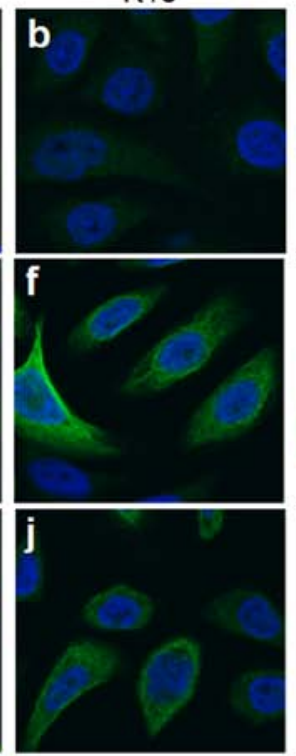

$\mathrm{K} 17$
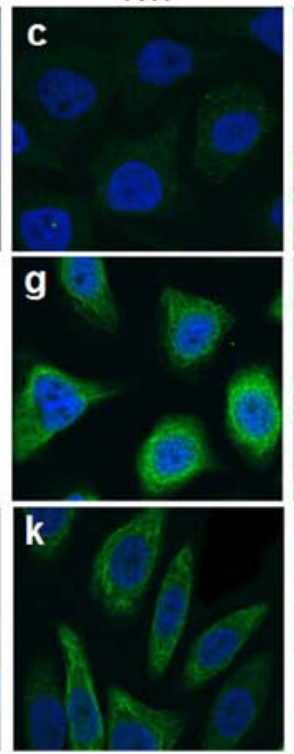

pan-K

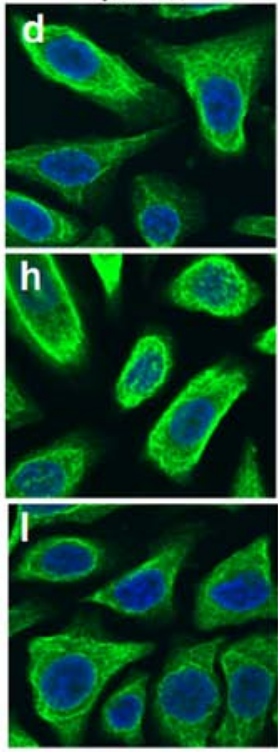

Figure 1. Expression of K6, K16 and K17 is increased in the cisplatin-resistant sublines of SNU601 cells. (A) SNU601 (WT), SNU601-cis2 (C2) and SNU601-cis10 (C10) cells were lysed, and equal amounts of cell extracts were analyzed by immunoblotting using antibodies against K6, K16, K17, pan-K, $\beta$-actin, and $\alpha$-tubulin. (B) SNU601 (WT) (a-d), SNU601-cis2 (C2) (e-h) and SNU601-cis10 (C10) (i-1) cells were reacted with antibodies against K6 (a, e and i), $\mathrm{K} 16$ (b, f and j), K17 (c, g and k), pan-K (d, h and 1), and stained with FITC-conjugated secondary antibody. Nuclei of the cells were stained with Hoechst 33342. Images were captured using a confocal microscope (magnification, x400; FV1000; Olympus). K, keratin.
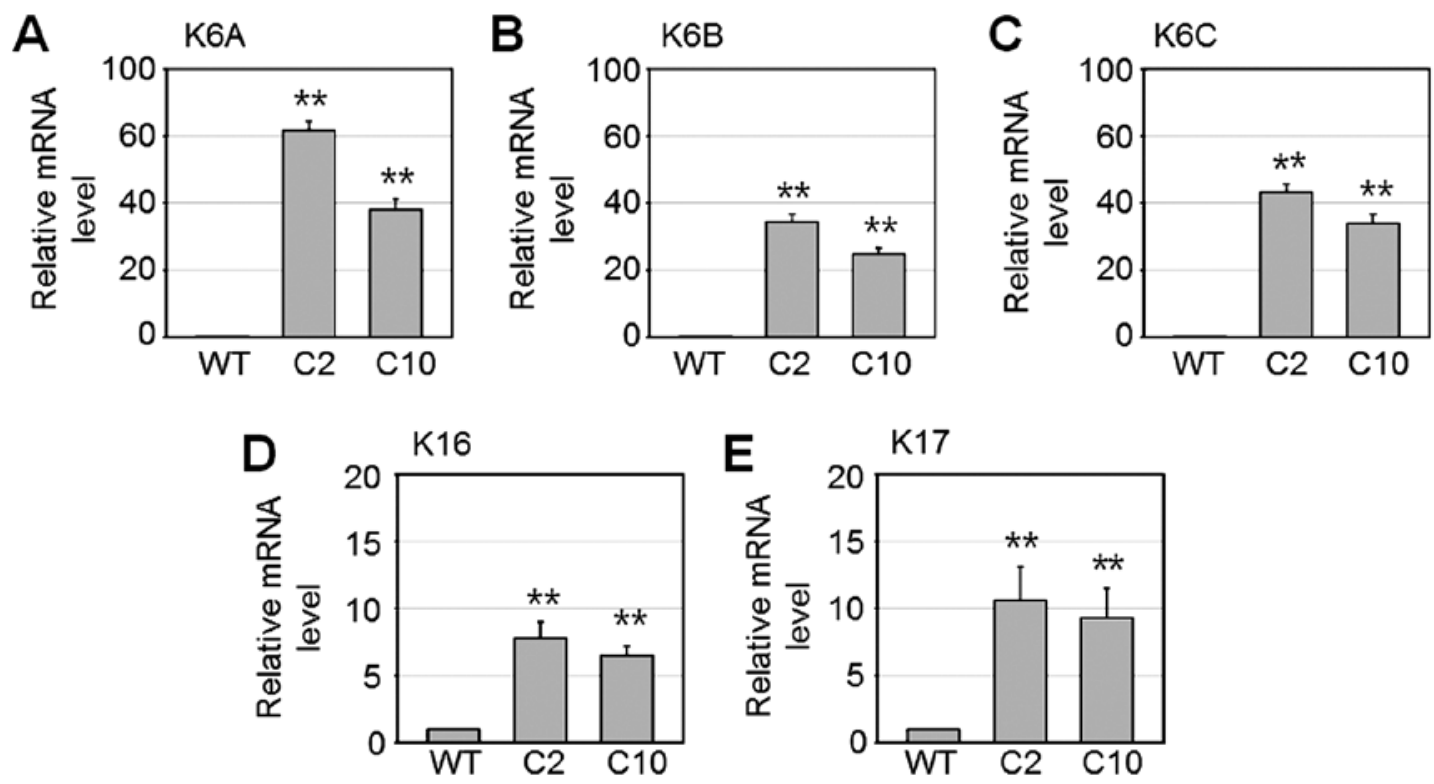

Figure 2. mRNA levels of $K 6 A, K 6 B, K 6 C, K 16$, and $K 17$ were upregulated in the cisplatin-resistant sublines of the SNU601 cells. SNU601 (WT), SNU601-cis2 (C2), and SNU601-cis10 (C10) cells were harvested and relative mRNA expression of $K 6 A(\mathrm{~A}), K 6 B(\mathrm{~B}), K 6 C$ (C), K16 (D), and $K 17$ (E) were analyzed by qPCR. ${ }^{* *} \mathrm{P}<0.001$ vs. WT cells. K, keratin.

(Fig. 3C and D). In agreement with this result, biochemical evidence also indicated that knockdown of K6 increased L-OHP-induced apoptosis in the resistant cells. Knockdown of K6 decreased the protein level of procaspase- 8 and increased levels of active caspase- 3 and cytoplasmic cytochrome $c$ in the SNU601-cis2 (C2) cells compared to the control RNA-transfected (CTLRNAi) cells, upon exposure to L-OHP (Fig. 3E). Based on this result, overexpression of $\mathrm{K} 6$ in the resistant cells appears to contribute, at least partially, to the acquisition of drug resistance.
Overexpression of K6A and K6B decreases drug responsiveness of wild-type cells. In the next experiment, to determine the effect of K6 on drug resistance, K6 expression was induced by introducing K6 overexpression vectors in the SNU601-WT cells and alteration in the cellular susceptibility was investigated. The cells were stably transfected with pCMV6-K6A and pCMV6-K6B, followed by selection with G418, and the expression of the introduced gene was confirmed by detection of the DDK-tag by immunoblotting (Fig. 4A). Then, control cells, and pCMV6-K6A- and pCMV6-K6B-expressing cells 
A

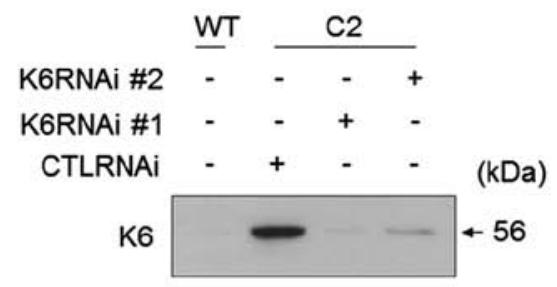

C

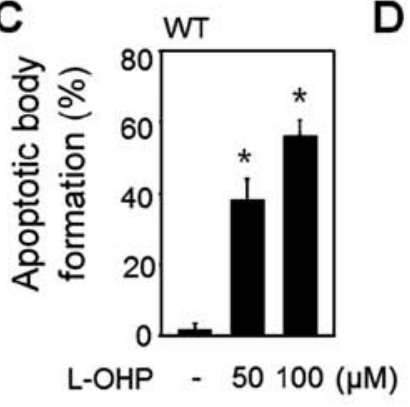

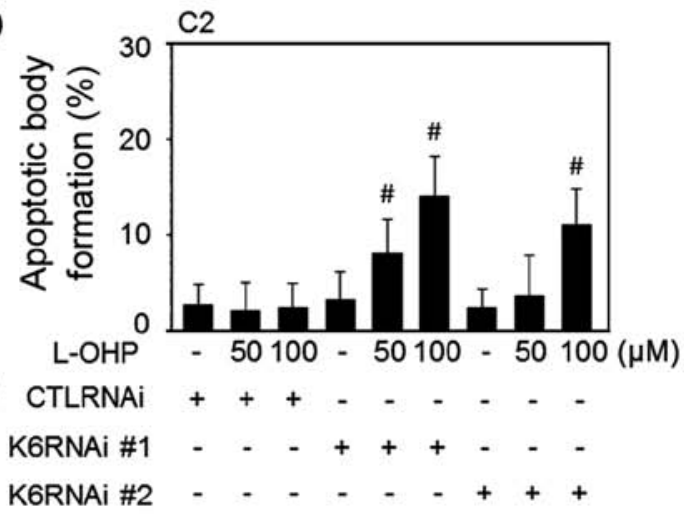

E

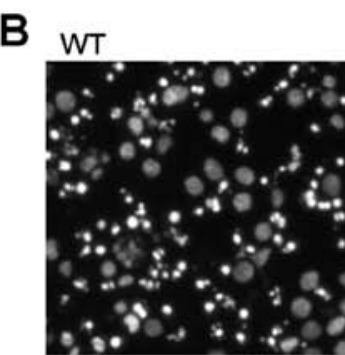

C2/K6RNAi \#1

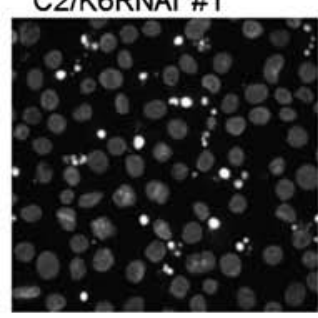

C2/CTLRNAi

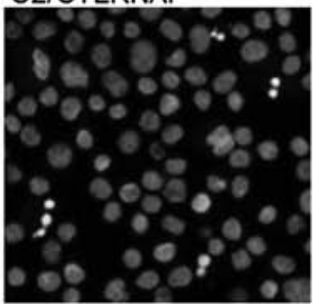

C2/K6RNAi \#2

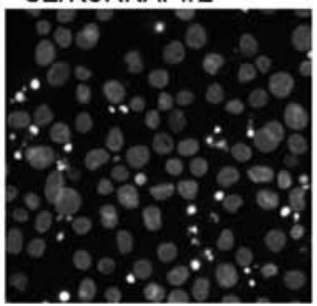

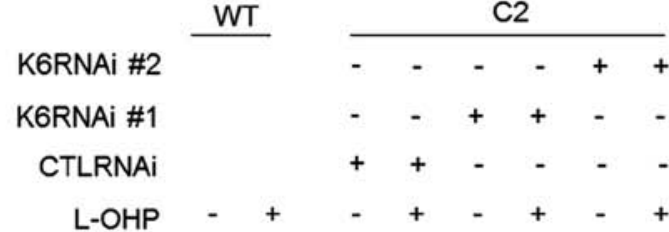

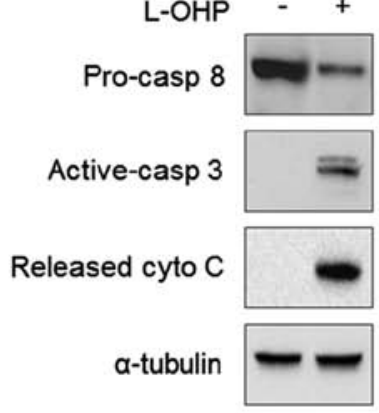

(kDa)
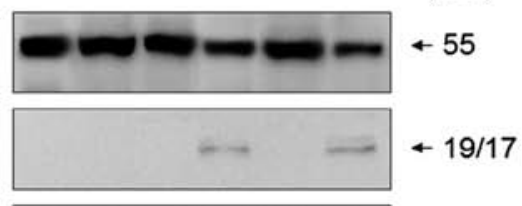

$\leftarrow 12$

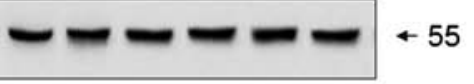

Figure 3. Knockdown of K6 partially restores the drug sensitivity of SNU601-cis2 cells. (A) SNU601 cells (WT) and SNU601-cis2 cells (C2), transfected with scrambled small interfering RNA (CTLRNAi), K6RNAi \#1 and K6RNAi \#2, were collected and the silencing effect of K6 was confirmed by immunoblot assay of cells not treated with L-OHP. (B-E) SNU601 cells (WT) and SNU601-cis2 cells (C2), transfected with scrambled small interfering RNA (CTLRNAi), K6RNAi \#1 and K6RNAi \#2, were exposed to $100 \mu \mathrm{M} \mathrm{L-OHP} \mathrm{for} 48 \mathrm{~h}$ (B and E), or 50 and $100 \mu \mathrm{M} \mathrm{L}-\mathrm{OHP}$ for $48 \mathrm{~h}$ (C and D). The treated cells were then subjected to apoptotic analysis (B-D) or immunoblotting to detect apoptotic proteins (E). For apoptosis analysis, the treated cells were stained with Hoechst 33342 and nuclear images were captured under a fluorescence microscope (B). Apoptotic body formation rates were calculated (C and D). For immunoblotting analysis, antibodies against pro-caspase- 8 , activated caspase-3, and cytochrome $\mathrm{c}$ were used. Total cell lysates were used to detect pro-caspase-8 (pro-casp-8), activated caspase-3 (active-casp-3) and non-mitochondrial cytosolic lysates were used to detect released cytochrome $c$ (released cyto $\mathrm{C}$ ) (E). ${ }^{*} \mathrm{P}<0.05$ vs. not treated cells; ${ }^{\prime} \mathrm{P}<0.05$ vs. CTLRNAi and L-OHP treated. $\mathrm{K}$, keratin; L-OHP, oxaliplatin.

were exposed to CDDP or L-OHP, and the percentages of apoptotic cells were detected. CDDP was less toxic than L-OHP and it induced apoptosis in $19.7 \%$ cells after a $48 \mathrm{~h}$ treatment at a concentration of $100 \mu \mathrm{M}$ in the control cells. On the other hand, the degree of apoptosis decreased to 11.2 and $13.6 \%$ in the K6A- and K6B-overexpressing cells, respectively (Fig. 4B). When treated with $30 \mu \mathrm{M}$ L-OHP, the control group exhibited $46.8 \%$ apoptotic cells, which was significantly higher than the percentage in the K6A- and K6B-overexpressing groups, which exhibited 26.1 and $29.5 \%$ apoptotic cells, respectively (Fig. 4C). Therefore, the increased expression of $\mathrm{K} 6$ seems to have a reducing effect on the reactivity to the drugs.

K6 overexpression inhibits lipid raft formation and localization of death receptor 5 (DR5). Platinum drugs have been shown to promote the extrinsic apoptotic pathway by forming lipid rafts and transferring death receptors into lipid rafts in cancer cells $(23,24)$. In the present study, we investigated whether this membrane event was affected by K6 expression in SNU601 cells. Lipid rafts can be stained using $\mathrm{CTxB}$, which exhibits specific affinity to ganglioside GM1, a component of lipid rafts. When SNU601-WT cells were exposed to L-OHP, lipid rafts visualized by CTXB-FITC were clearly observed at the cell membrane, indicating that lipid rafts were actively formed (Fig. 5A). However, in SNU601-cis 2 cells, FITC-stained lipid raft structure was barely detected. When K6 expression was suppressed in the resistant cells by gene interference using two types of siRNAs (C2/K6RNAi \#1 and C2/K6RNAi \#2), the size and frequency of fluorescence spots visible as lipid rafts increased compared to the control RNA-transfected resistant (C2/CTLRNAi) cells (Fig. 5A). Then, we separated the lipid raft fraction from whole cell lysates and detected the protein levels of DR5 and Fas-associated protein with death domain (FADD) by immunoblotting. Upon exposure to $100 \mu \mathrm{M}$ L-OHP, high levels of DR5 and FADD were detected in the raft fraction, indicating primary localization of DISC factors in the lipid rafts in the wild-type (WT) cells, but much less localization in lipid rafts in the resistant cells. However, the localization of DR5 and FADD in the lipid raft region was 


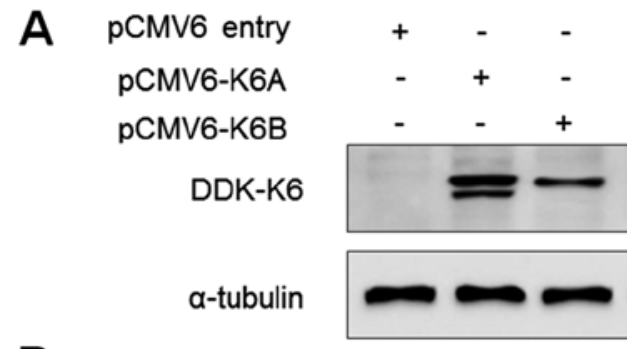

B

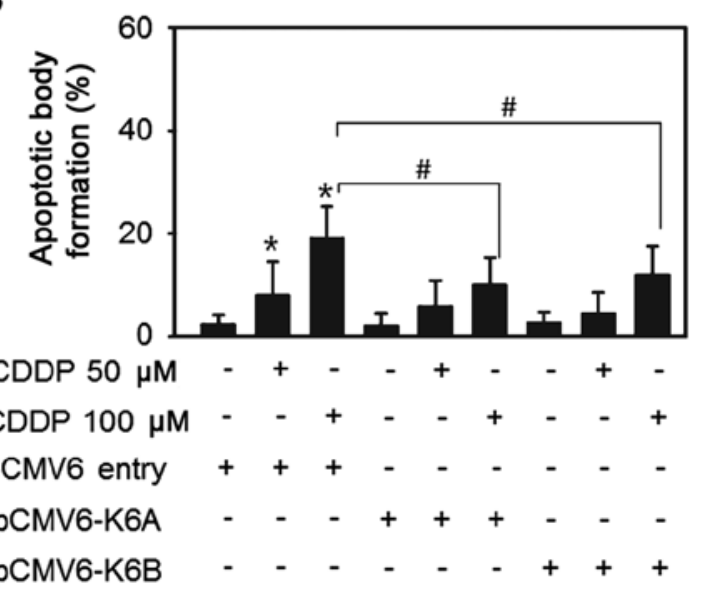

C

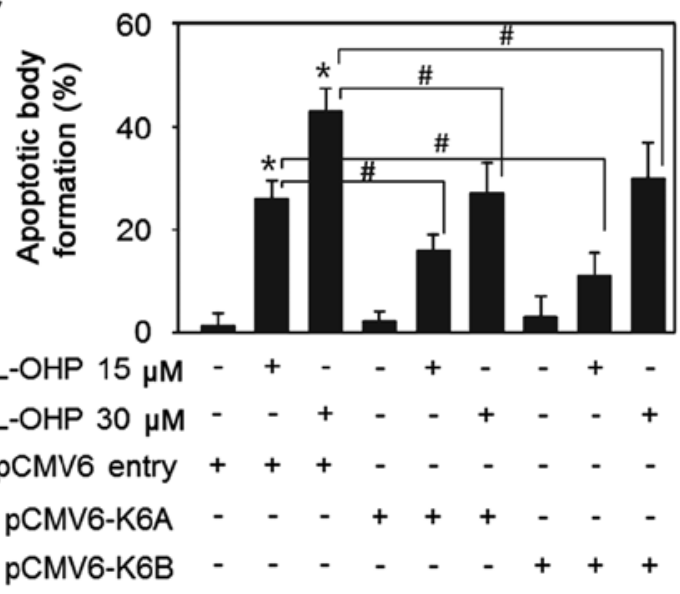

Figure 4. Introduction of K6 expression vectors reduced the drug responsiveness of SNU601 cells. SNU601-WT cells were stably transfected with pCMV6 entry (control), pCMV6-K6A, and pCMV6-K6B vectors. (A) The transfected cells were collected and total protein extracts were subjected to immunoblotting to detect DDK-tagged K6 expression. (B and C) The transfected cells were exposed to 50 and $100 \mu \mathrm{M}$ cisplatin (B) or 15 and $30 \mu \mathrm{M} \mathrm{L-OHP} \mathrm{(C)} \mathrm{for}$ $48 \mathrm{~h}$ and stained with Hoechst 33342. Nuclear images were captured under a fluorescence microscope and apoptotic body formation rates were calculated. ${ }^{*} \mathrm{P}<0.05$ vs. not treated; ${ }^{*} \mathrm{P}<0.05$ vs. pCMV6 entry and drug treated. CDDP, cisplatin; L-OHP, oxaliplatin.
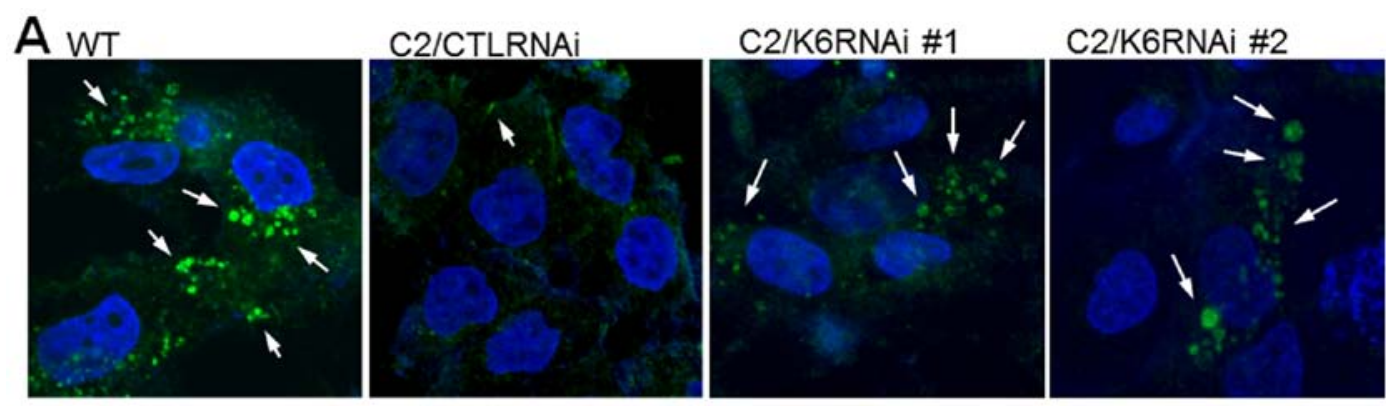

B

$\mathrm{C} 2$

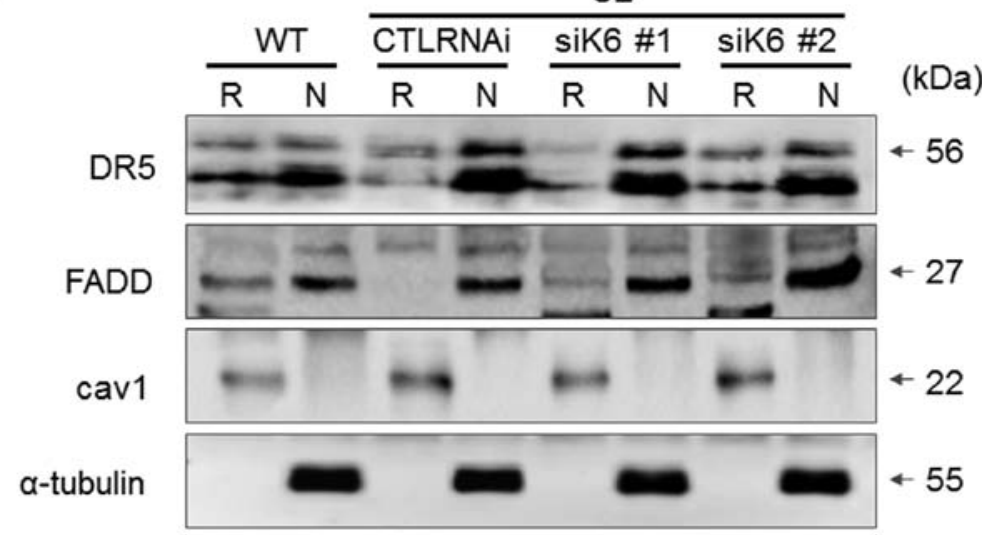

Figure 5. Knockdown of K6 in SNU601-cis2 cells restores lipid-raft structures and DR5 translocation into lipid raft fraction. (A) SNU601 (WT) and SNU601-cis2 cells (C2), transfected with scrambled small interfering RNA (C2/CTLRNAi), either K6RNAi \#1 or K6RNAi \#2, were exposed to $100 \mu \mathrm{M}$ L-OHP for $6 \mathrm{~h}$. After fixation, cells were stained with CTxB-FITC and observed under a confocal microscope (magnification, x400; FV1000; Olympus). Arrows indicate lipid-raft structures. (B) SNU601 (WT) and SNU601-cis2 cells (C2), transfected with scrambled small interfering RNA (CTLRNAi), either K6RNAi \#1 (siK6 \#1) or K6RNAi \#2 (siK6 \#2), were exposed to $100 \mu \mathrm{M} \mathrm{L-OHP} \mathrm{for} 24 \mathrm{~h}$ and lipid raft fractions or non-lipid raft fractions were obtained and analyzed by immunoblotting to detect DR5 and FADD. caveolin-1 (cav1), lipid raft loading control; $\alpha$-tubulin, non-raft protein loading control. R, raft; N, non-raft; L-OHP, oxaliplatin; DR5, death receptor 5; FADD, Fas-associated protein with death domain. 
higher in the K6 RNAi transfected SNU601-cis2 (siK6 \#1 and siK6 \#2) cells compared to the scrambled RNA transfected SNU601-cis2 (CTLRNAi) cells (Fig. 5B). Therefore, it was suggested that the increase in intracellular K6 in the resistant cells acts as a potential factor that decreases the lipid raft formation and inhibits translocation of death receptor and adaptor protein into lipid rafts.

\section{Discussion}

In the present study, it was demonstrated that the expression of specific keratin members, K6, K16 and K17, were significantly upregulated in the cisplatin-resistant variants of SNU601 gastric cancer cells compared to those in the parental cells. However, the overall intracellular keratin level was not significantly different in the resistant cells when pan-K was detected using western blot and immunofluorescence analyses, indicating that K6, K16, and K17 are specifically induced with little effect on other keratins. The expression of keratins is generally coordinated with specific partners of the opposite type. Thus, the increase in a keratin stimulates induction of the partner keratin, as well as the downregulation of a keratin leads to the degradation of its partner through ubiquitination. Among these three keratins, K6 is a type II keratin and the isoforms of K6 were shown as pairing partners with type I keratins, K16 and $\mathrm{K} 17$, enabling complete keratin filament formation. Here, we focused on the role of the most highly expressed keratin, $\mathrm{K} 6$, since it may represent the effects of K6/K16 and K6/K17.

In general, K6 filaments are characteristically expressed in hair follicles, oral epithelia, and palmoplantar epidermis (25). In addition to its constitutive expression, K6 is also elevated in the proliferative basal cells in wound edges of the skin where it appears to regulate cell growth in injured epithelial tissue during wound repair (26). K6/K16 and K6/K17 pairs are also elevated in certain skin diseases that induce keratinocyte hyperproliferation, such as psoriasis or skin tumors, and thus, it is frequently referred to as hyperproliferation- or activation-associated keratin $(27,28)$. Therefore, overexpression of K6 seems to be associated with the regulation of cell functions, such as proliferation or wound healing apart from simple structural and mechanical support. However, the significance of its induction in cancer has not yet been evaluated. Here, we modulated the expression of $\mathrm{K} 6$ to understand the role of the overexpression of K6 intermediate filaments. Gene silencing of K6 in the resistant cells restored anticancer drug-mediated apoptosis, as detected by enhancement of apoptotic body formation, caspase- 8 and -3 activation, and cytochrome $c$ release. With a similar tendency, overexpression of $\mathrm{K} 6 \mathrm{~A}$ and $\mathrm{K} 6 \mathrm{~B}$ vectors in the parental cells reduced apoptosis upon exposure to cisplatin and oxaliplatin. Hence, an elevated level of K6 appears to be associated with cellular resistance to apoptosis. To date, the association of other subtypes of keratin, especially K8, with resistance to chemotherapeutic drugs has been suggested in several studies. Hepatocytes from K8-null mice were found to be more sensitive to Fas-mediated apoptosis than their wild-type counterparts (29), and the role of $\mathrm{K} 8$ in the protection from apoptosis mediated by toxic agents was found to be related to c-FLIP content and ERK1/2 signaling (14). Transfection of K8 and K18 in mouse L cells and ectopic expression of K8/K18 in NIH3T3 fibroblasts led to resistance against multiple anticancer drugs $(30,31)$.
Suppression of K8 was found to be associated with the sensitivity of nasopharyngeal carcinoma cells to cisplatin (32). In addition, association of K5 overexpression with chemoresistance of ovarian cancer has been suggested (33). However, to the best of our knowledge, the present study is the first report on the role of $\mathrm{K} 6$ in drug tolerance.

The mechanisms by which K6 induces resistance in this system should be studied in more detail, but it seems to contribute to suppression of the extrinsic apoptotic pathway. We observed that K6 overexpression reduced lipid raft formation and the translocation of DR5 into the lipid raft fraction. In line with our results, there have been reports that the role of keratin in the protection from apoptosis is linked to the suppression of membrane events for death receptor activation. K8/K18 was shown to provide resistance to Fas-induced apoptosis and this protection occurred through modulation of Fas targeting to the cell surface in hepatocytes (29). Furthermore, $\mathrm{K} 8$ expression has been implicated in the regulation of the size of lipid rafts and the migration of Fas into lipid rafts through acid-sphingomyelinase activation $(34,35)$. Thus, suppression of the lipid raft-mediated apoptotic pathway seems to be one of the mechanisms involved in keratin-mediated cell resistance to cytotoxic agents. The results of our study suggest that K6 contributes to the cell resistance against cytotoxic stimuli by affecting death receptor redistribution into lipid rafts. The findings from this study suggest that keratins should be considered not only as cancer markers, but also as regulators of cancer cell signaling and drug responsiveness, and the fine-tuning of the regulatory mechanism of keratin expression may provide an effective strategy against chemotherapy-resistant cancer.

\section{Acknowledgements}

We thank Professor Cheol-Hee Choi for the cisplatin-resistant SNU601 cells and Ms. Jeong-Eun Choi and Ms. Yoo-Ri Choi for their excellent technical assistance.

\section{Funding}

This study was supported by a research fund from Chosun University, 2016.

\section{Availability of data and materials}

The datasets used during the present study are available from the corresponding author upon reasonable request.

\section{Authors' contributions}

SIH designed and wrote the manuscript. SCL and KRP performed the experiments and analyzed the data. SIH and SCL reviewed and edited the manuscript. All authors read and approved the final manuscript and agree to be accountable for all aspects of the research in ensuring that the accuracy or integrity of any part of the work are appropriately investigated and resolved.

\section{Ethics approval and consent to participate}

Not applicable. 


\section{Patient consent for publication}

Not applicable.

\section{Competing interests}

The authors declare that they have no competing interests.

\section{References}

1. Komatsu M, Sumizawa T, Mutoh M, Chen ZS, Terada K, Furukawa T, Yang XL, Gao H, Miura N, Sugiyama T and Akiyama S: Copper-transporting P-type adenosine triphosphatase (ATP7B) is associated with cisplatin resistance. Cancer Res 60: 1312-1316, 2000.

2. Cort A, Ozben T, Saso L, De Luca C and Korkina L: Redox control of multidrug resistance and its possible modulation by antioxidants. Oxid Med Cell Longev 2016: 4251912, 2016.

3. Wang J, Zhou JY and Wu GS: ERK-dependent MKP-1-mediated cisplatin resistance in human ovarian cancer cells. Cancer Res 67: 11933-11941, 2007.

4. Islam SU, Shehzad A, Sonn JK and Lee YS: PRPF overexpression induces drug resistance through actin cytoskeleton rearrangement and epithelial-mesenchymal transition. Oncotarget 8 : 56659-56671, 2017.

5. Foerster F, Braig S, Moser C, Kubisch R, Busse J, Wagner E, Schmoeckel E, Mayr D, Schmitt S, Huettel S, et al: Targeting the actin cytoskeleton: Selective antitumor action via trapping PKCvarepsilon. Cell Death Dis 5: e1398, 2014.

6. Mukhtar E, Adhami VM and Mukhtar H: Targeting microtubules by natural agents for cancer therapy. Mol Cancer Ther 13 $275-284,2014$

7. Xi J, Zhu X, Feng Y, Huang N, Luo G, Mao Y, Han X, Tian W, Wang G, Han X, et al: Development of a novel class of tubulin inhibitors with promising anticancer activities. Mol Cancer Res 11: 856-864, 2013.

8. Moll R, Divo M and Langbein L: The human keratins: Biology and pathology. Histochem Cell Biol 129: 705-733, 2008

9. Moll R, Franke WW, Schiller DL, Geiger B and Krepler R: The catalog of human cytokeratins: Patterns of expression in normal epithelia, tumors and cultured cells. Cell 31: 11-24, 1982.

10. Fuchs E: Keratins and the skin. Annu Rev Cell Dev Biol 11: 123-153, 1995

11. Coulombe PA and Omary MB: 'Hard' and 'soft' principles defining the structure, function and regulation of keratin intermediate filaments. Curr Opin Cell Biol 14: 110-122, 2002.

12. Galvin S, Loomis C, Manabe M, Dhouailly D and Sun TT: The major pathways of keratinocyte differentiation as defined by keratin expression: An overview. Adv Dermatol 4: 277-300, 1989.

13. Van Muijen GN, Warnaar SO and Ponec M: Differentiation-related changes of cytokeratin expression in cultured keratinocytes and in fetal, newborn, and adult epidermis. Exp Cell Res 171: 331-345, 1987.

14. Gilbert S, Loranger A and Marceau N: Keratins modulate c-Flip/extracellular signal-regulated kinase 1 and 2 antiapoptotic signaling in simple epithelial cells. Mol Cell Biol 24: 7072-7081, 2004.

15. Roux A, Loranger A, Lavoie JN and Marceau N: Keratin 8/18 regulation of insulin receptor signaling and trafficking in hepatocytes through a concerted phosphoinositide-dependent Akt and Rab5 modulation. FASEB J 31: 3555-3573, 2017.

16. Lahdeniemi IAK, Misiorek JO, Antila CJM, Landor SK Stenvall CA, Fortelius LE, Bergström LK, Sahlgren C and Toivola DM: Keratins regulate colonic epithelial cell differentiation through the Notch1 signalling pathway. Cell Death Differ 24: 984-996, 2017.

17. Srivastava SS, Alam H, Patil SJ, Shrinivasan R, Raikundalia S, Chaudhari PR and Vaidya MM: Keratin 5/14mediated cell differentiation and transformation are regulated by TAp63 and Notch1 in oral squamous cell carcinomaderived cells. Oncol Rep 39: 2393-2401, 2018
18. Chivu-Economescu M, Dragu DL, Necula LG, Matei L, Enciu AM, Bleotu C and Diaconu CC: Knockdown of KRT17 by siRNA induces antitumoral effects on gastric cancer cells. Gastric Cancer 20: 948-959, 2017.

19. Xu H, Choi SM, An CS, Min YD, Kim KC, Kim KJ and Choi $\mathrm{CH}$ : Concentration-dependent collateral sensitivity of cisplatin-resistant gastric cancer cell sublines. Biochem Biophys Res Commun 328: 618-622, 2005.

20. Livak KJ and Schmittgen TD: Analysis of relative gene expression data using real-time quantitative PCR and the 2(-Delta Delta C(T)) method. Methods 25: 402-408, 2001.

21. Iwasaki I, Sugiyama H, Kanazawa S and Hemmi H: Establishment of cisplatin-resistant variants of human neuroblastoma cell lines, TGW and GOTO, and their drug cross-resistance profiles. Cancer Chemother Pharmacol 49: 438-444, 2002.

22. Sun CL and Chao CC: Cross-resistance to death ligand-induced apoptosis in cisplatin-selected HeLa cells associated with overexpression of DDB2 and subsequent induction of cFLIP. Mol Pharmacol 67: 1307-1314, 2005.

23. Xu L, Qu X, Zhang Y, Hu X, Yang X, Hou K, Teng Y, Zhang J, Sada K and Liu Y: Oxaliplatin enhances TRAIL-induced apoptosis in gastric cancer cells by CBL-regulated death receptor redistribution in lipid rafts. FEBS Lett 583: 943-948, 2009.

24. Huang CR, Jin ZX, Dong L, Tong XP, Yue S, Kawanami T, Sawaki T, Sakai T, Miki M, Iwao H, et al: Cisplatin augments FAS-mediated apoptosis through lipid rafts. Anticancer Res 30: 2065-2071, 2010.

25. Rothnagel JA, Seki T, Ogo M, Longley MA, Wojcik SM, Bundman DS, Bickenbach JR and Roop DR: The mouse keratin 6 isoforms are differentially expressed in the hair follicle, footpad, tongue and activated epidermis. Differentiation 65: 119-130, 1999.

26. Wong P and Coulombe PA: Loss of keratin 6 (K6) proteins reveals a function for intermediate filaments during wound repair. J Cell Biol 163: 327-337, 2003.

27. Jiang CK, Magnaldo T, Ohtsuki M, Freedberg IM, Bernerd F and Blumenberg M: Epidermal growth factor and transforming growth factor alpha specifically induce the activation- and hyperproliferation-associated keratins 6 and 16. Proc Natl Acad Sci USA 90: 6786-6790, 1993.

28. Leigh IM, Navsaria H, Purkis PE, McKay IA, Bowden PE and Riddle PN: Keratins (K16 and K17) as markers of keratinocyte hyperproliferation in psoriasis in vivo and in vitro. Br J Dermatol 133: 501-511, 1995.

29. Gilbert S, Loranger A, Daigle N and Marceau N: Simple epithelium keratins 8 and 18 provide resistance to Fas-mediated apoptosis. The protection occurs through a receptor-targeting modulation. J Cell Biol 154: 763-773, 2001.

30. Bauman PA, Dalton WS, Anderson JM and Cress AE: Expression of cytokeratin confers multiple drug resistance. Proc Natl Acad Sci USA 91: 5311-5314, 1994.

31. Anderson JM, Heindl LM, Bauman PA, Ludi CW, Dalton WS and Cress AE: Cytokeratin expression results in a drug-resistant phenotype to six different chemotherapeutic agents. Clin Cancer Res 2: 97-105, 1996

32. Wang Y, He QY, Tsao SW, Cheung YH, Wong A and Chiu JF: Cytokeratin 8 silencing in human nasopharyngeal carcinoma cells leads to cisplatin sensitization. Cancer Lett 265: 188-196, 2008.

33. Ricciardelli C, Lokman NA, Pyragius CE, Ween MP, Macpherson AM, Ruszkiewicz A, Hoffmann P and Oehler MK: Keratin 5 overexpression is associated with serous ovarian cancer recurrence and chemotherapy resistance. Oncotarget 8 : 17819-17832, 2017

34. Gilbert S, Loranger A, Lavoie JN and Marceau N: Cytoskeleton keratin regulation of FasR signaling through modulation of actin/ezrin interplay at lipid rafts in hepatocytes. Apoptosis 17: 880-894, 2012.

35. Gilbert S, Loranger A, Omary MB and Marceau N: Keratin impact on PKCס- and ASMase-mediated regulation of hepatocyte lipid raft size-implication for FasR-associated apoptosis. J Cell Sci 129: 3262-3273, 2016. 